\title{
EDITORIAL
}

\section{PSMA PET-CT: the winner takes it all}

\author{
Massimo Lazzeri ${ }^{1,2} \cdot$ Arturo Chiti ${ }^{1,3}$
}

Published online: 25 August 2021

(c) The Author(s), under exclusive licence to Springer-Verlag GmbH Germany, part of Springer Nature 2021

In 2021, prostate, lung, and colorectal cancers (CRCs) will account for $46 \%$ of all incident cases in men, with prostate cancer (PCa) alone accounting for $26 \%$ of all diagnoses [1]. PCa has been the focus of much research in the last 2 decades with treatment advances, ranging from robot-assisted surgery to focal therapy and high-precision radiotherapy, with curative intent, to a growing number of new therapies for advanced disease [2], [3]. Molecular probes and imaging, particularly prostate-specific membrane antigen (PSMA) PET-CT, are likely to be increasingly important for PCa management.

In the current issue of European Journal of Nuclear Medicine and Molecular Imaging, Rüschoff and co-workers add a piece of evidence to the potential role of [68 Ga]-PSMA-11 PET-CT in PCa decision-making [4]. They correlated histopathological parameters and immunohistochemical PSMA expression patterns on radical prostatectomy specimens with [68 Ga]-PSMA-11 PET-CT. They investigated the WHO/ ISUP grade groups, growth pattern (expansive vs. infiltrative), tumor area, and diameter as well as immunohistochemical PSMA heterogeneity, intensity, and negative tumor area (PSMA \%neg), with imaging. Their findings suggest that patients with a high PSMA \%neg, infiltrative growth pattern, smaller tumor size, and WHO/ISUP grade group 2 may have a lower [68 Ga]-PSMA-11 uptake in prostate cancer. Authors must be praised for the excellent strategy, which

This article is part of the Topical Collection on OncologyGenitourinary.

Massimo Lazzeri

massimo.lazzeri@humanitas.it

1 Department of Biomedical Sciences, Humanitas University, Via Rita Levi Montalcini 4, 20090 Pieve Emanuele, Milan, Italy

2 Department of Urology, IRCCS Humanitas Research Hospital, via Manzoni 56, 20089 Rozzano, Milan, Italy

3 Department of Nuclear Medicine, IRCCS Humanitas Research Hospital, via Manzoni 56, 20089 Rozzano, Milan, Italy correlates findings on whole gland pathological samples with imaging. Linking the imaging with the whole prostate is the right method to represent the distribution of PSMA staining overcoming the bias of biopsy which represents only a minimal part of the gland. This study might have significant clinical implications supporting a role for PSMA PET in the primary diagnosis setting.

A vast range of new imaging modalities are being assessed as tools for decision-making in patients with suspected PCa [5].

Multiparametric MRI (mpMRI) has a large body of literature including several multi-sites clinical trials and [6] recognized by the EAU (European Association of Urology) as a useful imaging technique to identify and detect lesions over conventional TRUS and systematic biopsy protocols.

However, adoption of mpMRI has been slowed by the increased costs, requirement for specific radiological expertise, complex MRI/US fusion procedure, and by the fact that, although the negative predictive value (NPV) is very high, some potentially relevant cancers may be invisible [7]. Several studies showed that [68 Ga]-PSMA-11 PET might have a high diagnostic value for PCa primary diagnosis, as it was shown to identify clinically significant PCa [8], [9], [10]. Similarly to PIRADS for reporting mpMRI findings, a PSMA-RADS has been recently introduced, and in the coming years, it will be more and more the standard for reporting and compare results [11]. PET imaging has a further advantage as well. Conventional imaging using $\mathrm{CT}$ and bone scan has insufficient sensitivity when staging men with intermediate- and high-risk localized PCa. Hofman and co-workers performed a multicenter, two-arm, randomized study, aiming to compare conventional imaging with $\mathrm{CT}$ and bone scanning or [68 Ga]-PSMA-11 PET-CT for staging. They found that [68 Ga]-PSMA PET-CT is a suitable replacement for conventional imaging, providing superior accuracy in staging localized PCa [12]. Thus, thanks to the whole-body imaging modality used, [68 Ga]-PSMA-11 PET/CT encompasses diagnosis and staging at the same time.

Imaging techniques have no place in routine followup of PCa if PSA is not rising. Although EAU does not 
yet recommend it, one of the current clinical options is to consider PSMA radioligands PET/CT, when available, as a first-line imaging modality for follow-up [13]. This fact has important clinical implications as PSMA-targeted endoradiotherapy/radioligand therapy (PRLT) with small-molecule, urea-based agents labeled with the $\beta$-particle-emitting radionuclide lutetium-177 (177Lu) has been recently introduced for the treatment of castration resistant PCa. According to ClinicalTrials.gov, there are about 15 ongoing trials examining the role of 177Lu-labeled PSMA-targeted agents in CRPC, opening the doors to personalized theragnostic approach.

In conclusion, PSMA PET-CT will be increasingly important in treatment decision-making along the whole spectrum of PCa. In the future, we should be ready for a new scenario which will overcome the boarders of single medical specialties. New imaging and technologies for diagnosis, follow-up, and treatment are potentially scalable, relatively affordable, and available in high volume university hospitals. The challenge is to identify optimal strategies for deployment and implementation of personalized medicine: i.e. patient molecular profile and target therapies. Inevitably, these sorts of changes will require a lot of investments and deliver challenges for all healthcare systems but also present important opportunities to reshape and optimize care.

All of us involved in the management of PCa will seek to assess these diagnostic and treatment developments, determine what is likely to constitute the best approach in different healthcare settings, and make policy and clinical practice recommendations.

\section{Declarations}

Ethical approval Institutional Review Board approval was not required because the paper is an editorial.

Informed consent Not applicable.

Conflict of interest The authors declare no competing interests.

\section{References}

1. Siegel RL, Miller KD, Fuchs HE, Jemal A. Cancer Statistics, 2021. CA Cancer J Clin. 2021;71(1):7-33. https://doi.org/10. 3322/caac. 21654.
2. Lazzeri M, Guazzoni G. Focal therapy meets prostate cancer. Lancet. 2010;376(9746):1036-7.

3. Roumeguère T, Aoun F, Albisinni S, Mjaess G. Antibodies targeting prostate-specific membrane antigen positive prostate cancer: from diagnostic imaging to theranostics. Curr Opin Oncol. 2021. https://doi.org/10.1097/CCO.0000000000000767.

4. Ru Rüschoff JH, Ferraro DA, Muehlematter UJ, et al. What's behind ${ }^{68} \mathrm{Ga}$-PSMA-11 uptake in primary prostate cancer PET? Investigation of histopathological parameters and immunohistochemical PSMA expression patterns. Eur J Nucl Med Mol Imaging. 2021. https://doi.org/10.1007/s00259-021-05501-1.

5. Lopci E, Lughezzani G, Castello A, et al. PSMA-PET and microultrasound potential in the diagnostic pathway of prostate cancer. Clin Transl Oncol. 2021;23(1):172-8. https://doi.org/10.1007/ s12094-020-02384-w.

6. Mottet N, van den Bergh RCN, Briers E, et al. EAU-EANMESTRO-ESUR-SIOG Guidelines on prostate cancer-2020 update. Part 1: Screening, Diagnosis, and Local Treatment with Curative Intent. Eur Urol. 2021;79(2):243-62. https://doi.org/10.1016/j. eururo.2020.09.042.

7. Le JD, Tan N, Shkolyar E, et al. Multifocality and prostate cancer detection by multiparametric magnetic resonance imaging: correlation with whole-mount histopathology. Eur Urol. 2015;67:569-76.

8. Lopci E, Guazzoni G, Lazzeri M. 68Ga prostate-specific membrane antigen PET/CT for primary diagnosis of prostate cancer: complementary or alternative to multiparametric MR imaging. Radiology. 2018;287(2):725-6. https://doi.org/10.1148/radiol. 2017172607.

9. Hoffmann MA, Miederer M, Wieler HJ, et al. Diagnostic performance of 68Gallium-PSMA-11 PET/CT to detect significant prostate cancer and comparison with 18F-FEC PET/CT. Oncotarget. 2017;8(67):111073-83.

10. Donato P, Roberts MJ, Morton A, et al. Improved specificity with 68Ga-PSMA PET/CT to detect clinically significant lesions "invisible" on multiparametric MRI of the prostate: a single institution comparative analysis with radical prostatectomy histology. Eur J Nucl Med Mol Imaging. 2019;46:20-30.

11. Werner RA, Thackeray JT, Pomper MG, et al. Recent updates on molecular imaging reporting and data systems (MI-RADS) for theranostic radiotracers-navigating pitfalls of SSTR- and PSMAtargeted PET/CT. J Clin Med. 2019;8(7):1060.

12. Hofman MS, Lawrentschuk N, Francis RJ, et al. proPSMA study group collaborators. Prostate-specific membrane antigen PET-CT in patients with high-risk prostate cancer before curative-intent surgery or radiotherapy (proPSMA): a prospective, randomised, multicentre study. Lancet. 2020;395(10231):1208-16. https://doi. org/10.1016/S0140-6736(20)30314-7.

13. von Eyben FE, Soydal C, von Eyben R. [68)Ga-PSMA PET/ CT for patients with PSA relapse after radical prostatectomy or external beam radiotherapy. Diagnostics (Basel). 2021;11(4):622. https://doi.org/10.3390/diagnostics11040622.

Publisher's note Springer Nature remains neutral with regard to jurisdictional claims in published maps and institutional affiliations. 